\title{
Dialysis Membrane Reaction
}

National Cancer Institute

\section{Source}

National Cancer Institute. Dialysis Membrane Reaction. NCI Thesaurus. Code C114714.

A severe hypersensitivity-like reaction occurring during dialysis due to exposure to the dialysis membrane. 